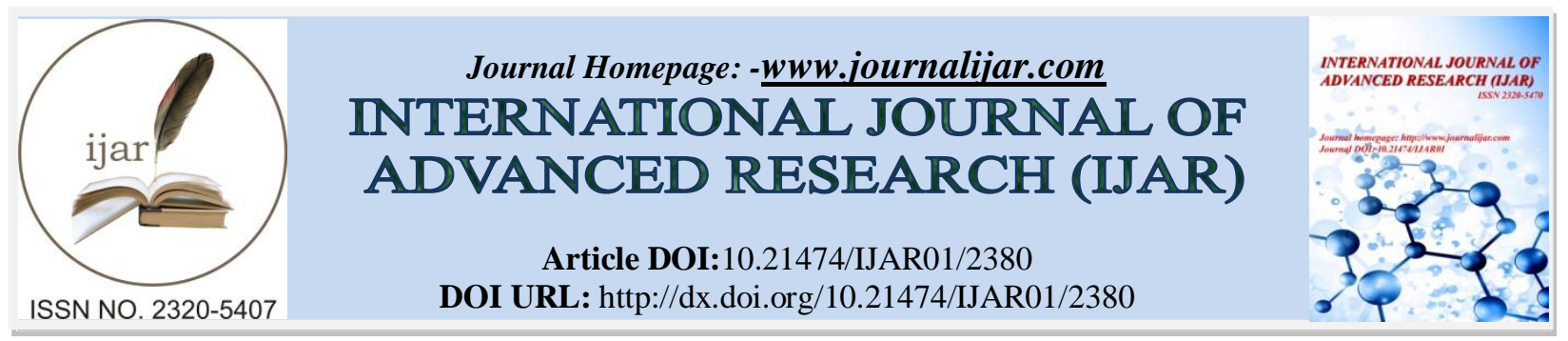

RESEARCH ARTICLE

\title{
RELATION BETWEEN FACIAL EXPRESSION AND EMOTION.
}

Samir Kumar Bandyopadhyay ${ }^{1}$ and Goutam Roy Chowdhury ${ }^{2}$.

1. Research Director, Techno India University Techno India University, EM-4, Sector-V, Salt Lake, Kolkata700091, West Bengal, India.

2. Chairman, Techno India Group Techno India University, EM-4, Sector-V, Salt Lake, Kolkata-700091, West Bengal, India.

\section{Manuscript Info}

Manuscript History

Received: 19 October 2016

Final Accepted: 20 November 2016

Published: December 2016

Key words:-

Facial Features, Facial Expressions, Support vector machine; multiclass classifier and human emotion.

\section{Abstract}

We use facial expressions not only to express our emotions, but also to provide important communicative cues during social interaction, such as our level of interest, ourdesire to take a speaking turn and a continuous feedback signaling about the understanding of theinformation conveyed. Among all the facial features, eye feature has more application domain. It is reportedthat facial expressions have considerable effects on listener, near about 55 percent effect of the spoken wordsdepend on eye movements and facial expressions of the speaker. Again facial expression has some relation with emotion. This paper studied the relation based on extraction of facial features and find out relation between them.

Copy Right, IJAR, 2016,. All rights reserved.

\section{Introduction:-}

Emotion is one type of affect i.e. temperament and sensation (for example, stain). Emotions can be viewed as either states or as processes. As a state (like being sad or joy), an emotion interacts with other mental states and causes certain behaviors. An emotion is a response to a specific stimulus either internal or from outside. Emotions often the stimulus by itself. Moods is caused by a specific stimulus. Emotions have brief duration than mood which has longer duration whereas moods last much longer.

Reading facial expressions of emotion can help credibility assessments Teachers can read the emotions of their students to obtain cues about the progress of their lesson plans so they can adjust accordingly and deliver them more effectively. Administrators read the emotions of their staff. Businesspersons and negotiators who can read the emotions of others can nurture mutually beneficial collaborations. Product researchers can improve the qualitative data they obtain from consumers by reading consumer's emotions when evaluating products, giving hints as to what they truly feel despite what they say about it. Parents, spouses, friends, and everyone with an interest in building strong and constructive relationships can benefit from improving their ability to read emotions.

People often get emotional when they lie, especially when the stakes are high. These emotions can occur because of the fear of getting caught, guilt or shame about the event lied about, or even because one likes the thought of successfully lying to others, especially those in positions of authority. Facial expressions can be signs of these emotions and the ability to detect them may be important for individuals working environment.

Corresponding Author:-Samir Kumar Bandyopadhyay.

Address:-Research Director, Techno India University Techno India University, EM-4, Sector-V, Salt

Lake, Kolkata-700091, West Bengal, India 
Many people can distinguish the emotions that are associated with different facial expressions. Even more, recent research has exposed that there may be more discernible categories of emotions than originally conceived to be seen through facial expressions. Until recently, scientists believed that there are six basic categories of emotion, including happiness, surprise, anger, sadness, fear, and disgust.

The analysis of the human face via image (and video) is one of the most interesting and focusing research topics in the last years for the image community. From the analysis (sensing, computing, and perception) of face images, much information can be extracted, such as the sex/gender, age, facial expression, emotion/temper, mentality/mental processes and behavior/psychology, and the health of the person captured. According to this information, many practical tasks can be performed and completed; these include not only person identification or verification (face recognition), but also the estimation and/or determination of person's profession, hobby, name (recovered from memory), etc. Research on face image analysis has been carried out and is being conducted around various application topics, such as (in alphabetical order) age estimation, biometrics, biomedical instrumentations, emotion assessment, face recognition, facial expression classification, gender determination, human-computer/humanmachine interaction, human behavior and emotion study, industrial automation, military service, psychosis judgment, security checking systems, social signal processing, surveillance systems, sport training, tele-medicine service, etc. Therefore facial expressions are the most important information for emotions perception in face to face communication.

The first part of the paper explains about an approach to the problem of facial feature extraction from an image. For face portion segmentation basic image processing operation like morphological dilation, erosion, reconstruction techniques with disk structuring element are used. Six permanent Facial features like eyebrows(left and right), eye (left and right), mouth and nose are extracted using facial geometry, edge projection analysis and distance measure and feature vector is formed considering height and width of left eye, height and width of left eyebrow, height and width of right eye, height and width of right eyebrow, height and width of nose and height and width of mouth along with distance between left eye and eyebrow, distance between right eye and eyebrow and distance between nose and mouth. Based on the features extracted next problem is to establish a relation between facial expression and emotion.

\section{Literature Review:-}

Human-like robots and machines that are expected to enjoy truly intelligent and transparent communications with human can be created using automatic facial expression recognition with a set of specific desired accuracy and performance requirements. Facial expression recognition deals with the problem of classifying facial images into expression classes and emotion is derived from expressions. It is of interest to a growing number of researchers and much progress has been made during the last two decades.

Expression recognition involves a variety of subjects such as perceptual recognition, machine learning, affective computing etc. One case study uses skin color range of human face to localize face area. Then high frequency noise is removed by masking with a low pass filter from the preprocessed image and skin color blocks are detected. After face detection, various facial features are identified by calculating the ratio of width of multiple regions in human face. Finally the test image is partitioned into a set of sub-images and each of these sub-images is matched against a set of sub-pattern training set. Given as input any emotion of face, this pattern training set will classify the particular emotion [1]. Face component extraction by dividing the face region into eye pair and mouth region and measurement of Euclidean distance among various facial features is also adopted by a case study. Similar study is done by Neha Gupta to detect emotions. This research includes four steps: pre-processing, edge detection, feature extraction and distance measurement among the features to classify different emotions. This type of approach is classified as Geometric Approach [2].

Another research includes Face detection method using segmentation technique. First, the face area of the test image is detected using skin color detection. RGB color space is transformed into YCbCr color space in the image and then skin blocks quantization is done to detect skin color blocks. As next step, a face cropping algorithm is used to localize the face region. Then, different facial features are extracted usingsegmentationof each component region (eyes, nose, mouth). Finally, vertical \& angular distances between various facialfeatures are measured and based on this any unique facial expression is identified. This approach can be usedin any biometric recognition system [3].A template matching based facial feature detection technique is used in a different case study [4].Different methods of 
face detection and their comparative study are done in another review work. Facedetection methods are divided into two primary techniques: Feature based \& View based methods [5].

Gabor filters are used to extract facial features in another study. This approach is called Appearance basedapproach. This classification based facial expression recognition method uses a bank of multilayerperceptron neural networks. Feature size reduction is done by Principal Component Analysis (PCA) [6]. Another study represents a robust face detection and gender classification strategy in color images undernon-uniform background. This is done by localizing human face region in the given image and detectingfacial features after converting the given RGB image to $\mathrm{YCbCr}$ color space for skin detection. Here, firstmouth region is detected from the localized face region. From this, eye regions and nose regions are locatedand they are used as feature points. Gender classification is done using these features on images withdifferent sizes. Linear support vector machine is used as the classifier which gives best classification rate [7].

Currently, most researchers have moved away from views of recognition as an automatic emotion detection process. Thus, "recognition" is regarded as a more complex inferential process with direct or indirect links to emotion [8-9].

\section{Image Enhancement;-}

In RGB images each pixel has a particular color; that color is described by the amount of red, green and blue in it. If each of these components has a range $0-255$, this gives a total of $256^{\wedge} 3$ different possible colors. Such an image is a "stack" of three matrices; representing the red, green and blue values for each pixel. This means that for every pixel there correspond 3 values. Whereas, in greyscale each pixel is a shade of gray, normally from 0 (black) to 255 (white). This range means that each pixel can be represented by eight bits, or exactly one byte. Other greyscale ranges are used, but generally they are a power of 2 . So, we can say gray image takes less space in memory in comparison to RGB images. The enhancement is done by the following ' $R$ ' Program. The results are shown in Figure 1 to Figure 4.
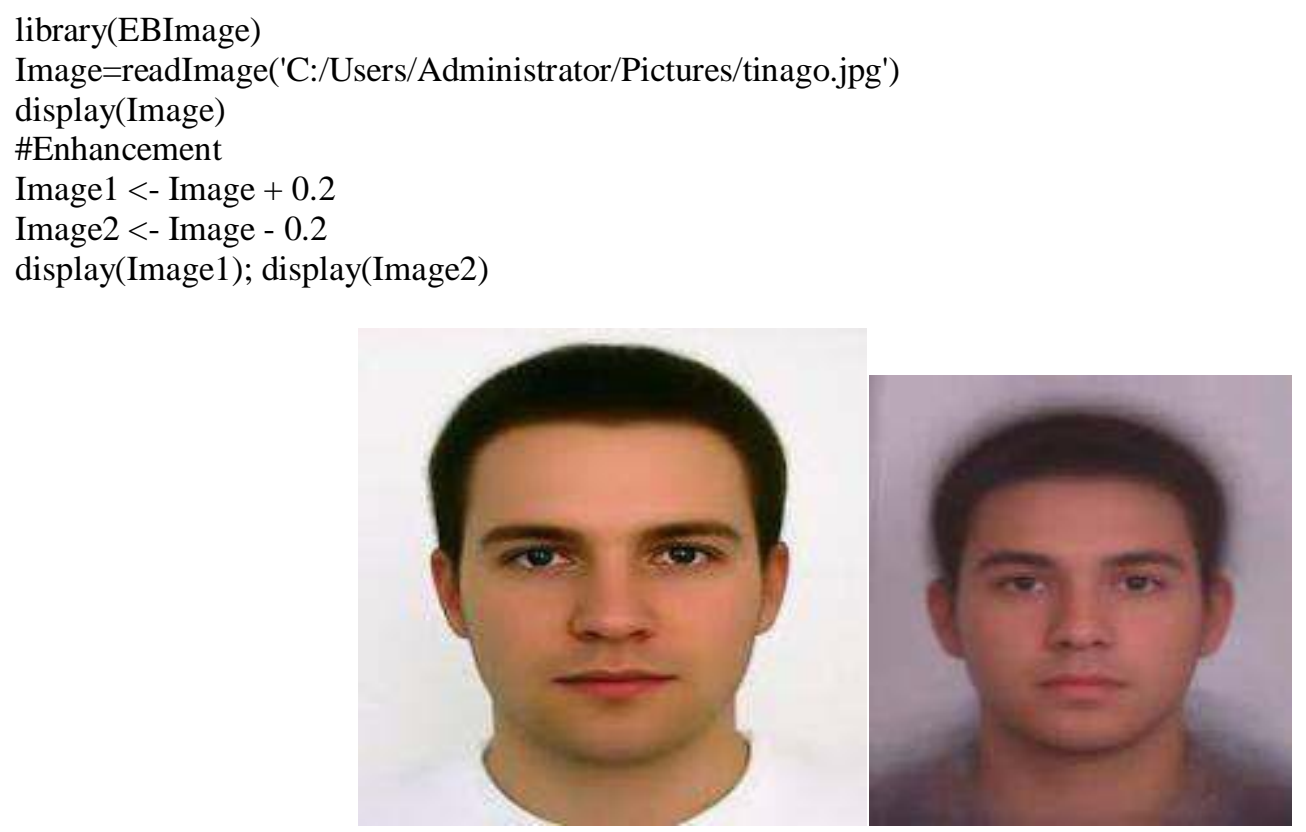

Fig.1:- Test image

Fig. 2:- Templates of this image. 


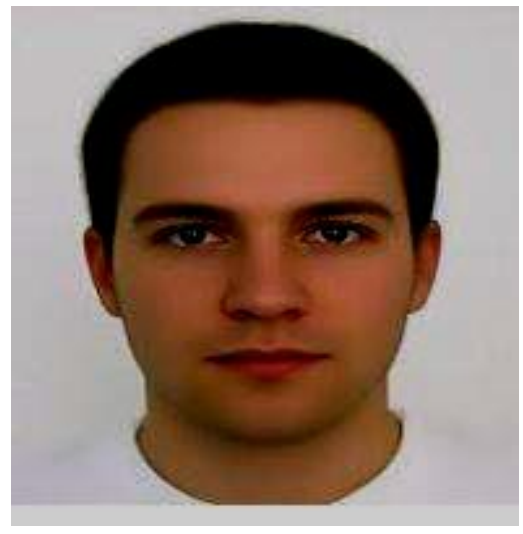

Fig. 3:- less Enhancement

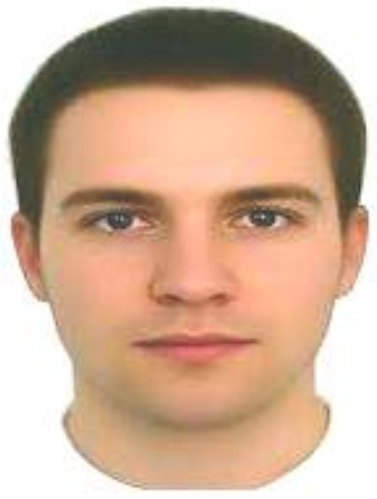

Fig.4:- More Enhancement

Face Detection:-

In this paper, we focus on only machine learning methods because they eliminate subjective thinking factors from human experience. Moreover, they only depend on training data to make final decisions. Thus, if training data is well organized and adequate, then these systems will achieve high performance without human factors.

\section{Face Alignment:-}

The face alignment is one of the important stages of the face recognition. Moreover, face alignment is also used for other face processing applications, such as face modeling and synthesis. Its objective is to localize the feature points on face images such as the contour points of eye, nose, mouth, and face.

\section{Facial Feature Extraction:-}

Face area and facial feature plays an important role in facial expression recognition. Better the feature extraction rate more is the accuracy of facial expression recognition. Precise localization of the face plays an important role in feature extraction, and expression recognition. But in actual application, because of the difference in facial shape and the quality of the image, it is difficult to locate the facial feature precisely.The output obtained after using procedures B-D is shown in Figure 5.

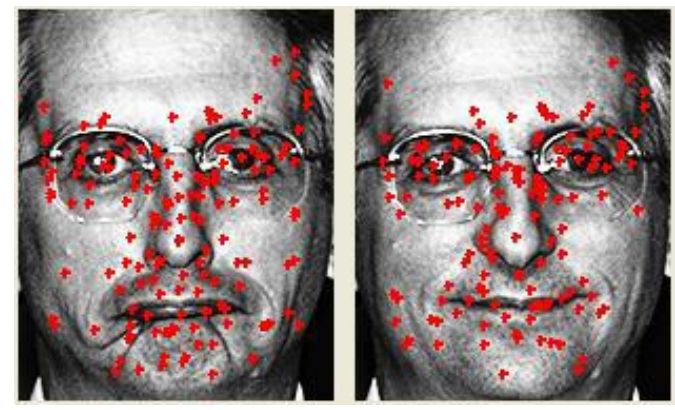

Fig 5:-Interest points in face image.

\section{Formation of Feature Vector:-}

Bounding box location of feature segments obtained in the above step are used to calculate the height and width of left eyebrow, height and width of left eye, height and width of right eyebrow, height and width of right eye, height and width of nose and height and width of mouth. Distance between centre of left eye and eyebrow, right eye and eyebrow and mouth and nose is also calculated. Thus total 15 parameters are obtained and considered as feature vector:

Thus,

$\mathrm{Fv}=\{\mathrm{H} 1, \mathrm{~W} 1, \mathrm{H} 2, \mathrm{~W} 2, \mathrm{H} 3, \mathrm{~W} 3, \mathrm{H} 4, \mathrm{~W} 4, \mathrm{Hn}, \mathrm{Wn}, \mathrm{Hm}, \mathrm{Wm}, \mathrm{D} 1, \mathrm{D} 2, \mathrm{D} 3\}$

Where,

$\mathrm{H} 1=$ height of left eyebrow, W1= width of left eyebrow

$\mathrm{H} 2=$ height of left eye, $\mathrm{W} 2=$ width of left eye

$\mathrm{H} 3=$ height of right eyebrow, W3= width of right eyebrow

$\mathrm{H} 4=$ height of right eye, $\mathrm{W} 4=$ width of right eye 
$\mathrm{Hn}=$ height of nose, $\mathrm{Wn}=$ width of nose,

$\mathrm{Hm}=$ height of mouth, $\mathrm{Wm}=$ width of mouth

D1 = distance between centre of left eyebrow and left eye,

$\mathrm{D} 2=$ distance between centre of right eyebrow and right eye,

D3= distance between centre of nose and mouth

The proposed algorithm can be described as:

1. Read input image

2. Image enhancement: Converting RGB image to Gray Scale

3. Face Detection using Adaptive Boosting and Artificial Neural Network

4. Face Alignment using Convolution Neural Network (CNN)

5. Crop the Aligned Face Image

6. Resize

7. Extract features from cropped face

Expressions Classification:-

Classification of available data sets can be viewed as the task of separating classes in features space. Inmachine learning, Support Vector Machines (SVMs) [10] are supervised learning models with associatedlearning algorithms that analyze data and recognize patterns used for classification. Given a set oftrainingexamples, each marked for belonging to one of two categories, an SVM training algorithm builds a modelthat assigns new examples into one category or the other, making it a non-probabilistic binary linearclassifier.

In the proposed algorithm, linear binary support vector machine model is used as multiclass classifier. Four different types of facial expressions of human face need to be classified according to the algorithm. As SVM is a binary classifier, the algorithm uses "one against all" approach to use SVM as multiclass classifier.

The algorithm uses four facial expressions:

- Neutral

- Smiling

- Angry

$\cdot$ Sad

The proposed work uses Database for face images for training set [11-12]. Each type of expression is presented by four images. The 16 images are arranged into three train database. First database contains all the 16 images of four types of expression. Second train database contains 12 images of Smiling, Angry \& Sad expressions. Third train database contains 8 images of Angry \& Sad expressions. These three training databases are used jointly to train the whole set of images.

First, preprocessing is done on the training set of images. Preprocessing of the training images and test images is needed because the used images are of different sizes and in RGB color space and they need to be of same features so that classification can be done accurately.

Preprocessing includes:

- The face images in the training set are converted to "double" type.

- The images are converted to gray scale from RGB color space.

- The images are zoomed or shrank to 200X200 pixels size.

- The images are reshaped so that the 2D matrices of the gray scale images are converted to $1 \mathrm{D}$ array

- of 40000 columns.

After image preprocessing, the three train databases are assigned different classes. The steps include:

- In the first training set, there are 12 images of which first four are of neutral expression. This expression is assigned class 1 and left 9 images are assigned class -1 .

- In the second training set, there are 9 images of which first four are of smiling expression. This expression is assigned class 2 and left 6 images are assigned class -2 .

- In the third set, there are 6 images of which first four are of angry expression assigned class 3 and

- $\quad$ left four are of sad expression assigned class -3. 
- Finally, the test image is mapped to either neutral class or smiling class or angry class or sad class and the same is displayed in the user interface.

The proposed algorithm are given below:

Impreprocess (Image_Pixel_Array)

Step1. Start

Step2. Convert Image_Pixel_Array to its corresponding double format letImage_Pixel_Array_Double.

Step3. If Image_Pixel_Array_Double is of format $a^{*} b^{*} 3$, thenconvert it to Corresponding Gray scale and save the pixel values to a $2 \mathrm{D}$ array letgImage.

Elsesave the pixel values of the input image to a 2D array let gImage.

Step4. Resize gImage to a Predefined size say $\mathrm{m} * \mathrm{n} \&$ save the pixel values to a 2D array letgImage_Resized.

Step5. Reshape gImage_Resized Array to a $2 \mathrm{D}$ array of size $1 *(\mathrm{mn}) \&$ save the pixel values to a2D array let gImage_Reshaped.

Step6. Return the Array gImage_Reshaped.

Step7. End

SVM_Training (Training_Data, Group_Membership_Class)

Step1. Start

Step2. Train Linear Support Vector Machine with Training_Data and Group_Membership_Class

and store the value in an array let SVM1.

Step3. Return the array SVM1

Step4. End

SVM_Classify (SVM_Trained, Img_Array)

Step1. Start

Step2. Classify Img_Array in one of the classes with SVM_Trained and SVM Binary Classifier

and store the value in a variable let Classifier 1

Step3. Return the value Classifier 1

Step4. End

Based on the above classifier the expression can be Neutral/Smiling/Angry/Sad.

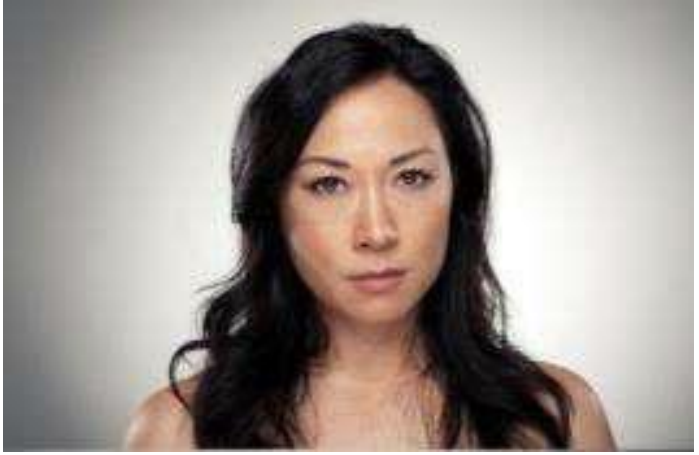

Fig. 6:- Neutral face

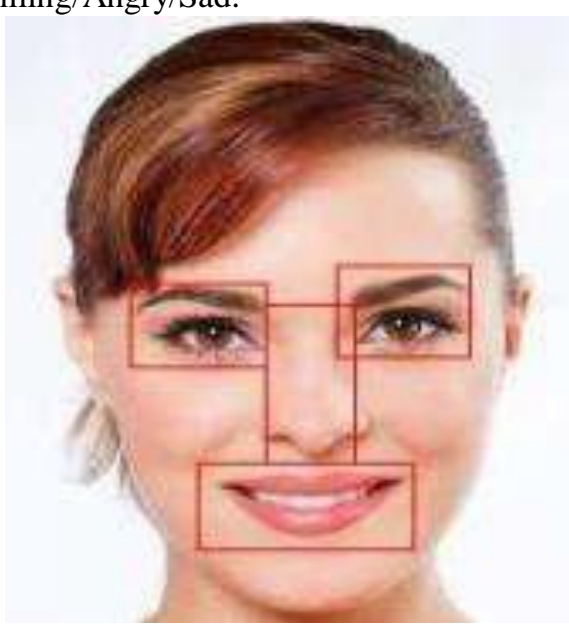

Fig.7:- Smiling face

\section{Conclusions:-}

This paper studied computationally efficient algorithm for facial feature selection based on templatematching method which further leads to identification of smiling face or neutral face. At first, minimalpre-processing including gray scale conversion is done on the image. After that, matching betweenoriginal image and template image is done using normalized cross-correlation technique. Each matchingarea is bounded by box to identify that region of interest. Then the mid points between the eye regions arefound and the distance between the mid points and the corners of the mouth region is calculated. On thebasis of the distances between these features, emotions are 
recognized. After detecting neutral or smilingface, different types of facial expressions are classified using linear support vector machine used asmulticlass classifier.

\section{References:-}

1. Alom Md. Zahangir, Piao Mei-Lan, Alam Md. Ashraful, Kim Nam, Park Jae-Hyeung. Facial

2. Expressions recognition from complex background using face context and adaptively weighted subpatternPCA" on World Academy of Science. Engineering and Technology. 2012;6.

3. DewiAgushinta R, AdangSuhendra, SarifuddinMadenda, Suryadi HS. Face component extractionusing segmentation method on face recognition system. On Journal of engineering Trends inComputing\& Information Science. 2011;2:2.

4. Gupta Neha, Kaur Prof. Navneet. Design and implementation of emotion recognition system by usingmatlab. On International Journal of Engineering Research and Applications (IJERA). 2013;3(4) :2002-2006.

5. Roy Sunita, Prof. Bandyopadhyay Samir Kumar. Extraction of facial features using a simple templatebased method. On International Journal of Engineering Science and Technology (IJEST).

6. Roy Sunita, Roy Sudipta, Prof. Bandyopadhyay Samir K. A tutorial review on face detection. OnInternational Journal of Engineering Science and Technology (IJEST). 2012;1(8).

7. Lajevardi SM, Lech M. Facial expression recognition using neural networks and log-gaborfilters.Digital Image Computing: Techniques and Applications IEEE; 2008.

8. Ravi S, Wilson S. Face Detection with facial features and gender classification based on supportvector machine. Special Issue - International Journal of Imaging Science and Engineering2010.

9. Matsumoto, D., Frank, M. G., \& Hwang, H. S. (2013). Nonverbal communication: science and applications. London, UK: SAGE.

10. Lindquist, K. A., Gendron, M., Barrett, L. F., \& Dickerson, B. C. (2014). Emotion perception, but not affect perception, is impaired with semantic memory loss.Emotion, 14, 375-387.

11. Ravi S, Wilson S. Face Detection with facial features and gender classification based on supportvector machine. Special Issue - International Journal of Imaging Science and Engineering2010.

12. Gross R, Face Databases, Handbook of Face Recognition, Stan Z. Li, Anil K. Jain, ed. Springer-Verlag. $2005 ; 22$. 